\title{
BMJ Open Observational study protocol for evaluating control of hypertension and the effects of social determinants
}

\author{
Heather Angier, ${ }^{1}$ Nathalie Huguet, ${ }^{1}$ Miguel Marino, ${ }^{1}$ Beverly Green, ${ }^{2}$ \\ Heather Holderness, ${ }^{1}$ Rachel Gold, ${ }^{3}$ Megan Hoopes, ${ }^{3}$ Jennifer DeVoe ${ }^{1}$
}

To cite: Angier $\mathrm{H}$, Huguet $\mathrm{N}$, Marino M, et al. Observational study protocol for evaluating control of hypertension and the effects of social determinants. BMJ Open 2019;9:e025975. doi:10.1136/ bmjopen-2018-025975

- Prepublication history for this paper is available online. To view these files, please visit the journal online (http://dx.doi org/10.1136/bmjopen-2018025975).

Received 28 August 2018 Revised 4 February 2019 Accepted 7 February 2019

Check for updates

(c) Author(s) (or their employer(s)) 2019. Re-use permitted under CC BY-NC. No commercial re-use. See rights and permissions. Published by BMJ.

${ }^{1}$ Family Medicine, Oregon Health \& Science University, Portland, Oregon, USA

${ }^{2}$ Research, Kaiser Permanente Washington Health Research Institute, Seattle, Washington, USA

${ }^{3}$ Research, OCHIN, Portland, Oregon, USA

Correspondence to Ms Heather Holderness; holdernh@ohsu.edu

\section{ABSTRACT}

Introduction Hypertension is a common chronic health condition. Having health insurance reduces hypertension risk; health insurance coverage could improve hypertension screening, treatment and management. The Medicaid eligibility expansion of the Affordable Care Act was ruled not to be required by the US Supreme Court. Subsequently, a 'natural experiment' was produced with some states expanding Medicaid eligibility while others did not. This presents a unique opportunity to learn whether and to what extent Medicaid expansion can affect healthcare access and services for patients at risk for and diagnosed with hypertension, and patients with undiagnosed hypertension. Additionally, social determinants of health (SDH), at both the individualand community-level, influence diagnosis and care for hypertension and it is important to understand how they interact with health insurance coverage changes.

Methods/design We will use electronic health record (EHR) data from the Accelerating Data Value Across a National Community Health Center Network clinical data research network, which has data from community health centres in 22 states, some that did and some that did not expand Medicaid. Data include information on changes in health insurance, service receipt and health outcomes from 2012 through the most recent data available. We will include patients between the ages of 19 and 64 years $(n=1$ 524241 ) with $\geq 1$ ambulatory visit to a community health centre. We will estimate differences in outcomes using difference-in-difference and difference-in-difference-indifference approaches. We will test three-way interactions with insurance group, time and social determinants of health factors to compare the potential effect of gaining insurance on our proposed outcomes.

Ethics and dissemination This study uses secondary data analysis and therefore approval for consent to participate was waived. The Institutional Review Board for OHSU approved this study. Approval reference number is: IRB00011858. We plan to disseminate our findings at relevant conferences, meetings and through peer-reviewed journals.

Trial registration number NCT03545763.

\section{BACKGROUND}

Hypertension is the most common chronic health condition among adults in the USA. About 80 million US adults (1 in 3) have

\section{Strengths and limitations of this study}

- Unbiased health record data

- Largest sample of community health center data in the USA.

- Rigorous and standardised statistical analyses.

- Limited by potential of some missing data.

hypertension ${ }^{1}$ and the prevalence is projected to increase an additional $10 \%$ by $2030 .{ }^{2}$ Hypertension is a risk factor for heart disease and stroke, ${ }^{3}$ yet only half of adults with hypertension have blood pressure (BP) readings in a healthy range (uncontrolled $\mathrm{BP}$ is the primary clinical signifier of hypertension). ${ }^{1}$

Having health insurance reduces hypertension risk, as insurance impacts access to needed preventive care. ${ }^{4}$ Uninsured patients are also less likely to receive regular hypertension screening, ${ }^{5}$ creating delays in receipt of screening and other preventive care that can lead to negative hypertension outcomes. Therefore, increasing patients' access to care-via health insurance coverage-could improve rates of hypertension screening, treatment and management. ${ }^{6}$

In addition to health insurance, it is likely that social determinants of health $(\mathrm{SDH})$ also influence hypertension diagnosis and receipt of preventive care,${ }^{7-9}$ and the impact of many individual-level $\mathrm{SDH}$ on hypertension is known. For example, there are racial/ethnic disparities in hypertension diagnosis rates, ${ }^{9}$ and low socioeconomic status and unemployment are associated with high hypertension rates. ${ }^{10}$ Evidence is conflicted, however, on whether access to healthcare mitigates the impact of such individual-level SDH factors on health outcomes. ${ }^{11}$ Community-level SDH (eg, availability of healthy food, safety, walkability, ${ }^{7}$ racial segregation ${ }^{9}$ and neighbourhood poverty level ${ }^{12}$ ) are also associated with hypertension rates. Yet, very little is known about how community-level SDH influence 
the pathway between health insurance coverage and healthcare usage and/or health outcomes. Patients with hypertension must conduct daily management activities (eg, taking medications and adhering to diet and exercise regimens) and both uninsurance and suboptimal SDH can create barriers to successful hypertension care and management. ${ }^{6}$ Understanding this relationship will identify potentially modifiable SDH that can lead to targeted interventions and tailored treatment plans. Since health insurance alone does not guarantee access to care or improved health outcomes, it is essential to account for patients' social and economic environment when assessing the impact of gaining healthcare insurance on hypertension care as SDH are likely to moderate the impact.

Healthcare reform efforts improved access to healthcare at the same time as national recommendations have prompted primary care providers to track and act on patients' SDH. Specifically, the Affordable Care Act (ACA) was enacted to improve access to healthcare and reduce health disparities ${ }^{13}$ by expanding Medicaid eligibility to adults earning $\leq 138 \%$ of the federal poverty level (FPL). Since the expansion was not required, as of March 2018, 32 states (and District of Columbia) implemented the expansion and 18 did not. ${ }^{14}$ This created a natural experiment to test the impact of gaining health insurance on hypertension prevalence, screening, treatment and management. Simulated models predicted the ACA would improve health outcomes and reduce disparities for patients with hypertension, ${ }^{15}$ yet actual results are not available to confirm or deny these predictions. In addition, the National Academy of Medicine, the American Academy of Pediatrics and the American Academy of Nursing ${ }^{16-18}$ all recommend tracking and using SDH in primary care settings to address health disparities. However, currently little is known about how access to patients' SDH information impacts hypertension care, especially in the context of changing health insurance availability. Thus, our quasi-experimental study will build on current understanding of how health insurance impacts hypertension diagnosis, detection, treatment and management, by comparing states that did versus did not expand Medicaid as part of the ACA, while assessing the potential moderating influence of patients' SDH status on the relationship between insurance coverage and these hypertension measures.

\section{METHODS}

\section{Study aims}

Our study will compare hypertension incidence, prevalence, screening, treatment (eg, medication use), and management (eg, hypertension control, systolic and diastolic BP change, risk factors related to hypertension control) in states that expanded Medicaid eligibility to those that did not before and after the ACA policy change. We will also assess individual-level and community-level
SDH that might moderate this relationship. The aims of this study are to:

\section{Aim 1}

Compare hypertension incidence, prevalence of undiagnosed hypertension, and rates of hypertension screening, in Medicaid expansion versus non-expansion states before and after the ACA.

\section{Aim 2}

Compare hypertension treatment (eg, medication use), and management (eg, hypertension control, systolic and diastolic BP change, risk factors related to hypertension control) in Medicaid expansion versus non-expansion states before and after the ACA.

\section{Aim 3}

Assess the extent to which rates of hypertension incidence, screening, and treatment effectiveness among patients who gained insurance versus those continuously insured or uninsured, pre-ACA/post-ACA, are moderated by individual-level SDH (eg, race, ethnicity), in expansion states.

\section{Aim 4}

Explore the interaction between community-level SDH (eg, neighbourhood racial segregation and deprivation) and hypertension incidence, screening, treatment and management among patients who gained insurance relative to those who were continuously insured or uninsured, in expansion states.

\section{Dataset}

Because many persons affected by Medicaid expansions are seen at community health centres (CHCs), our nation's healthcare 'safety net,' we will use data from the Accelerating Data Value Across a National Community Health Center Network (ADVANCE) clinical data research network (CDRN) of the National Patient-Centered Clinical Research Network (PCORnet). The ADVANCE CDRN includes individual- and community-level patient data from CHCs in 22 states (some that did and some that did not expand Medicaid). The ADVANCE CDRN aggregates CHC data from OCHIN, Health Choice Network, and geocoded community-level SDH data into one central research-ready data warehouse, hosted by OCHIN. Details about the ADVANCE CDRN can be found elsewhere. ${ }^{19}$

CHCs are required to collect and report many individual-level SDH data to the US Health Resources and Services Administration (HRSA). Therefore, the ADVANCE CDRN has data on race/ethnicity, language, FPL, homelessness and family size on nearly all patients. The ADVANCE CDRN also includes appended geography-based community-level SDH data for each patient. The profile of patients in the ADVANCE CDRN is comparable to national estimates of CHC patient characteristics with regard to poverty and insurance status. ${ }^{20}$ 


\section{Inclusion/exclusion criteria}

Our study period will range from 1 January 2012 to 31 December 2016 to allow for understanding the short-term, medium-term and long-term impacts of the ACA Medicaid expansion on hypertension prevalence, screening, treatment and management. Specific study periods will be defined for each analysis depending on the outcome and research question. The number of states will vary based on inclusion/exclusion criteria for each analysis. For example, if the inclusion criteria include a 24-month pre-ACA period the analytic dataset will include nine expansion and four non-expansion states. The study sample will include patients from the ADVANCE CDRN between the ages of 19 and 64 years $(\mathrm{n}=1524241)$, as these are the patients most likely affected by ACA-Medicaid expansions. Additionally, for clinic-level analyses, we will require $\geq 1$ visit pre-ACA or $\geq 1$ visit post-ACA, while for patient-level analyses, we will require $\geq 1$ visit pre-ACA and $\geq 1$ visit post-ACA. We will exclude pregnant women because there is a Medicaid programme that covers low-income pregnant women not otherwise eligible for Medicaid and pregnant women have very different usage patterns and healthcare needs than non-pregnant women.

\section{Measure definitions}

\section{Medicaid expansion}

We will define the pre-Medicaid and post-Medicaid expansion periods based on if and when a state expanded Medicaid. The initial implementation of the ACAMedicaid expansion took effect on 1 January 2014. While other states adopted the expansion later. Thus, pre-Medicaid / post-Medicaid expansion time periods may be different and/or states may cross over from non-expansion to expansion states depending on the analysis.

\section{Hypertension and undiagnosed hypertension}

We will use the guidelines for high BP using those that were recommended at the time of the visit, as this is what providers would have based their hypertension diagnosis on.

\section{Hypertension}

We will identify patients with a hypertension diagnosis using the following International Classification of Disease (ICD)codes: ICD-9: 401.00-401.99, 402.00-405.99 or ICD-10: I10-I15.

\section{Undiagnosed hypertension}

We will use a combination of visits with elevated BP, absence of a hypertension diagnosis and lack of medication prescribed to identify undiagnosed hypertension. ${ }^{21}$ If $\mathrm{BP}$ is elevated at two or more clinic visits, and no diagnostic codes were used to document why a diagnosis of hypertension was not made (ie, ICD-10 R03.0: white coat syndrome without hypertension or ICD-9 796.2: elevated $\mathrm{BP}$ without hypertension), high BP is likely undiagnosed hypertension. This will also be confirmed by an absence of antihypertensive medications.

\section{Insurance status}

Since health insurance status is based on information specific to payer types collected at each visit for billing purposes, it is a reliable source of information on coverage status at each visit. We will use previously developed algorithms ${ }^{22}$ to define insurance status as follows:

- Gained insurance (newly insured): uninsured at all visits in the pre-period and had all visits in the post-period paid by Medicaid or private (we may separate this category by insurance type [Medicaid or private] depending on the analysis).

- Continuously insured: all visits paid by Medicaid or private in both the pre-periods and post-periods (we may separate this category by insurance type [Medicaid or private] depending on the analysis).

- Continuously uninsured: no coverage for all visits in both the pre-periods and post-periods.

\section{Outcome measures}

Primary outcome measures will include rates of:

- Hypertension diagnosis.

- Undiagnosed hypertension.

- Hypertension screening using BP measurement.

- Hypertension treatment, including antihypertension medications.

- Hypertension management based on national recommendations during the study period (eg, Healthcare Effectiveness Data and Information $\mathrm{Set}^{23}$ ).

Secondary outcome measures include rates of coverage, insurance status, usage (rates and types of healthcare visits), preventive service receipt and hypertension-related complications (eg, incidence of cardiovascular [CVD] disease).

Clinic-level covariates (eg, clinic size, patient population characteristics and so on) will be included as needed and relevant for specific analyses.

Individual-level SDH will include age, race/ethnicity, household income, language preference, homelessness, family size, veteran status, migrant status, rurality and hypertension and CVD risk factors. ${ }^{24} \mathrm{CHCs}$ are required to collect and report many of these individual-level SDH data to HRSA to receive funding or designation under the Health Center Program.

Community-level SDH geocoded in the ADVANCE database conceptualised to potentially impact hypertension include, but are not limited to, data elements shown in table 1 and derived from US Census, American Community Survey, US Department of Agriculture Food Access Research Atlas, and other publicly available geospatial data sources. ${ }^{25}$ To assess the heterogeneity of the effect of Medicaid expansion on hypertension outcomes by community-level SDH, we hypothesise, for example, that there will be a greater reduction in hypertension levels and/or increase in proportion of patients with controlled hypertension among newly insured patients (compared with continuously insured patients) in communities with greater social and economic resources relative to their counterparts in less resourced communities. 


\begin{tabular}{|c|c|}
\hline $\begin{array}{l}\text { Community-level } \\
\text { SDH category }\end{array}$ & Metrics \\
\hline Built environment & $\begin{array}{l}\text { Fast food restaurants per } 100000 \\
\text { population }\end{array}$ \\
\hline $\begin{array}{l}\text { Race/ethnic } \\
\text { composition }\end{array}$ & $\begin{array}{l}\text { Count and per cent by race } \\
\text { Residential segregation (dissimilarity, } \\
\text { exposure) }\end{array}$ \\
\hline $\begin{array}{l}\text { Neighbourhood } \\
\text { resources }\end{array}$ & $\begin{array}{l}\text { Modified retail food environment } \\
\text { index (no of healthy food stores } \\
\text { divided by all food stores) } \\
\text { Per cent of people in a county living } \\
\text { more than } 1 \text { mile from a supermarket } \\
\text { or large grocery store if in an urban } \\
\text { area, or more than } 10 \text { miles if in a } \\
\text { rural area } \\
\text { Percentage of population living within } \\
1 / 2 \text { mile of a park } \\
\text { Recreation facilities per } 100000 \\
\text { population } \\
\text { Urban Classification Code }\end{array}$ \\
\hline $\begin{array}{l}\text { Neighbourhood } \\
\text { socioeconomic } \\
\text { composition }\end{array}$ & $\begin{array}{l}\text { Per cent of population with bachelor's } \\
\text { degree or higher } \\
\text { - Median household income } \\
\text { Per cent below } 100 \% \text { of federal } \\
\text { poverty level (FPL); per cent below } \\
200 \% \text { of FPL } \\
\text { - Unemployment rate }\end{array}$ \\
\hline $\begin{array}{l}\text { Social deprivation } \\
\text { index }\end{array}$ & $\begin{array}{l}\text { Composite measure of social } \\
\text { deprivation validated to be more } \\
\text { strongly associated with poor } \\
\text { access to healthcare and poor health } \\
\text { outcomes than a measure of poverty } \\
\text { alone }\end{array}$ \\
\hline
\end{tabular}

\section{Statistical analysis}

We will summarise baseline measures using descriptive statistics and data visualisation methods to characterise data across patient, clinic, and state groups. Our primary criteria for studying Medicaid expansion will be estimating differences in outcomes in expansion versus non-expansion states up to 24 months pre-Medicaid and at least 24 months post-Medicaid expansion using a difference-in-differences (DD) and a difference-in-difference-in-difference (DDD) approach. ${ }^{26}$ The DD and DDD approaches account for potential secular effects and changing policies that would affect both expansion and non-expansion states over time, while adjusting for potential confounders. Moreover, the DD and DDD approaches allow adjustment for differences between states in the pre-period. We will use General Linear or Non-Linear Mixed Effects Models, ${ }^{27}$ which offer a wide range of parametric distributions to model the dependent variables. To model continuous hypertension biomarkers over time (including looking at linear, quadratic and other types of trends), we will consider growth curve models to account for different timing and frequency of biomarker measurements. ${ }^{28}$

For Aims 1 and 2, the primary independent variable is whether a patient is in a state that expanded Medicaid or not. For Aims 3 and 4, the primary independent variable is whether a patient is newly insured, continuously insured or uninsured. We will use the continuously insured as the control group for these analyses. Interaction terms between the primary independent variable and time will be included in the model to assess the impact of the ACA Medicaid expansion on rate of hypertension incidence, prevalence, screening, diagnosis, treatment and management. Additionally, we will test three-way interactions with insurance group, time and $\mathrm{SDH}$ factors to compare the potential effect of gaining insurance on our proposed outcomes by SDH characteristic.

To account for significant patient, clinic and community differences in expansion and non-expansion states, we will use propensity score methods to reduce the observed bias, help minimise external threats to the validity of the results and adjust for imbalances between patient and clinic groups. We will use multilevel propensity score weighting ${ }^{29}$ to create similar comparison groups for all aims. We will include patient-, clinic- and statelevel data in our propensity score models and address performance of propensity score models through balance checks and standardised differences. We plan to implement bootstrapping and permutation tests to obtain reliable SEs when appropriate and to assess the robustness of our inference. Our multistate, multiyear design allows us the flexibility to incorporate and account for any potential health reform changes that may occur during our longitudinal study.

\section{Patient and public involvement}

No patients nor the public were involved in the development of the research question. No patient advisers will be included in this research project.

\section{DISCUSSION}

Healthcare access, which is highly dependent on having continuous health insurance, influences the likelihood of receiving hypertension care ${ }^{6}$ Having health insurance is also associated with improved health outcomes ${ }^{30}$; lack of insurance is associated with hypertension underdiagnosis and poor management. ${ }^{6}$ The ACA's Medicaid expansion, optional by state, created an opportunity to understand the impact of gaining health insurance on hypertension incidence, screening, treatment and management. Prior studies of individual states' Medicaid expansions showed that such expansion increased healthcare service usage, receipt of recommended preventive care and improved health outcomes, ${ }^{31}$ but these studies were limited by having no 'control' states for comparison. The 2014 Medicaid expansion created a 'natural experiment' for assessing whether and how gaining insurance coverage improves hypertension prevention and care. 
Individual- and community-level SDH are also known to negatively impact hypertension care ${ }^{7-9}$ and likely affect the relationship between health insurance and receiving hypertension care. Yet almost nothing is known about the moderating effect of SDH at either level on the relationship between health insurance and hypertension management and treatment. Our study will provide information with substantial implications for health policymakers at the local and national levels. In addition, we will assess the moderating impact of SDH on hypertension treatment and care, among people with and without insurance. As recommendations to track and act on $\mathrm{SDH}$ in primary care continue to increase, this study will also help answer questions about the interplay between health insurance and SDH.

\section{Strengths and limitations}

Our study has several limitations. We are unable to identify patients who died during the study time period, as the ADVANCE EHR data does not include information on mortality. We also anticipate some missing data, either from services documented in inaccessible parts of the EHR (likely random) or from patients who went outside the CHCs included in the ADVANCE CDRN to receive services (perhaps not random). However, evidence suggests that primary care providers outside of CHCs are not accepting or are significantly limiting the number of Medicaid-insured patients in their panels. ${ }^{32} 33$ Moreover, uninsured CHC patients who obtain Medicaid post-ACA are likely to continue receiving healthcare from $\mathrm{CHCs},{ }^{22}$ and our analyses can accommodate missing data resulting from attrition of patients. We will explicitly model missingness by including related variables in the analysis as covariates. ${ }^{34}$ If non-trivial levels of missing data are observed, we will use multiple imputation methods developed for big data to include these patients in analyses. ${ }^{35}$ As with any real-world study, unobserved changes might occur over time, making it difficult to isolate the effect of the ACA-Medicaid expansion or other health policy changes. Our approach is strengthened by propensity score weighting to account for differences between expansion and non-expansion states and the use of DD and DDD approaches to account for and minimise these biases. We will also conduct relevant sensitivity analyses.

\section{CONCLUSION}

This project will increase understanding of how the ACA Medicaid expansion impacted hypertension incidence, screening, treatment and management. It will also identify SDH that are barriers or facilitators to hypertension management among patients. Identifying potentially modifiable SDH that improve hypertension management may lead to targeted interventions. Community-level SDH data can help clinics conduct outreach in specific neighbourhoods or allow for EHR tools that link neighbourhood and patient data to inform clinical care and provide community resources. Such interventions could also provide tailored treatment plans based on individualand/or community-level SDH. Additionally, identifying healthcare behaviour after gaining insurance can help providers guide their patients toward on-time screening, medication adherence, exercise and diet change to improve hypertension management.

Contributors JD is $\mathrm{Pl}$ and led the writing of the grant application, with input from $\mathrm{NH}, \mathrm{HA}, \mathrm{RG}$ and BG. MM was responsible for biostatistics analysis planning. HA led the writing of the protocol manuscript with significant contributions from JD, NH, MM, BG, HH, RG and MH. All authors read and approved the final manuscript.

Funding This work was supported by the National Lung, Heart, and Blood Institute, grant number R01HL136575. ADVANCE (Accelerating Data Value Across a National Community Health Center Network) is led by the OCHIN Community Health Information Network in partnership with the Health Choice Network (HCN), Fenway Health, Care Oregon, Kaiser Permanente Center for Health Research, Legacy Health, Oregon Health and Science University (OHSU) and the Robert Graham Center. This study was made possible by the National Heart, Lung, and Blood Institute Grant \#R01HL136575.

\section{Competing interests None declared.}

Patient consent for publication Not required.

Ethics approval This study uses secondary data analysis and therefore approval for consent to participate was waived. The Institutional Review Board for Oregon Health \& Science University approved this study. Approval reference number is: IRB00011858. We plan to disseminate our findings at relevant conferences, meetings and through peer-reviewed journals.

Provenance and peer review Not commissioned; externally peer reviewed.

Open access This is an open access article distributed in accordance with the Creative Commons Attribution Non Commercial (CC BY-NC 4.0) license, which permits others to distribute, remix, adapt, build upon this work non-commercially, and license their derivative works on different terms, provided the original work is properly cited, appropriate credit is given, any changes made indicated, and the use is non-commercial. See: http://creativecommons.org/licenses/by-nc/4.0/.

\section{REFERENCES}

1. Centers for Disease Control and Prevention. High blood pressure fact sheet nd. http://www.cdc.gov/dhdsp/data_statistics/fact_sheets/fs_ bloodpressure.htm

2. Heidenreich PA, Trogdon JG, Khavjou OA, et al. Forecasting the future of cardiovascular disease in the United States: a policy statement from the American Heart Association. Circulation 2011;123:933-44.

3. Rapsomaniki E, Timmis A, George J, et al. Blood pressure and incidence of twelve cardiovascular diseases: lifetime risks, healthy life-years lost, and age-specific associations in $1 \cdot 25$ million people. Lancet 2014;383:1899-911.

4. Decker SL, Kostova D, Kenney GM, et al. Health status, risk factors, and medical conditions among persons enrolled in Medicaid vs uninsured low-income adults potentially eligible for Medicaid under the Affordable Care Act. JAMA 2013;309:2579-86.

5. Ayanian JZ, Weissman JS, Schneider EC, et al. Unmet health needs of uninsured adults in the United States. JAMA 2000;284:2061-9.

6. Centers for Disease Control and Prevention (CDC). Vital signs: prevalence, treatment, and control of hypertension--United States, 1999-2002 and 2005-2008. MMWR Morb Mortal Wkly Rep 2011;60:103-8.

7. Mujahid MS, Diez Roux AV, Morenoff JD, et al. Neighborhood characteristics and hypertension. Epidemiology 2008;19:590-8.

8. Mozaffarian D, Benjamin EJ, Go AS, et al. Heart disease and stroke statistics-2016 update: a report from the american heart association. Circulation 2016;133:e38-e360.

9. Mujahid MS, Diez Roux AV, Cooper RC, et al. Neighborhood stressors and race/ethnic differences in hypertension prevalence (the Multi-Ethnic Study of Atherosclerosis). Am J Hypertens 2011;24:187-93.

10. Levenstein S, Smith MW, Kaplan GA. Psychosocial predictors of hypertension in men and women. Arch Intern Med 2001;161:1341-6.

11. Lasser KE, Himmelstein DU, Woolhandler S. Access to care, health status, and health disparities in the United States and Canada: 
results of a cross-national population-based survey. Am J Public Health 2006;96:1300-7.

12. Booth JM, Jonassaint CR. The role of disadvantaged neighborhood environments in the association of John Henryism with hypertension and obesity. Psychosom Med 2016;78:552-61.

13. Robert Wood Johnson Foundation. How does the Affordable Care Act address racial and ethnic disparities in health care? Issue Brief. Princeton, N.J, 2011.

14. The Henry J Kaiser Family Foundation. Status of state action on

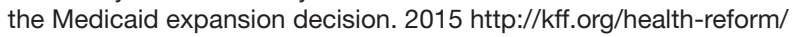
state-indicator/state-activity-around-expanding-medicaid-under-theaffordable-care-act/ (Accessed 27 Sep 27 2016)

15. Li S, Bruen BK, Lantz PM, et al. Impact of health insurance expansions on nonelderly adults with hypertension. Prev Chronic Dis 2015;12:E105.

16. Institute of Medicine. Capturing social and behavioral domains and measures in electronic health records: phase 2. Washington, DC: The National Academies Press, 2014.

17. Council on Community Pediatrics. Committee on Native American Child Health. Policy statement-health equity and children's rights. Pediatrics 2010;125:838-49.

18. Troseth MR. American academy of nursing endorses social behavioral determinants of health in electronic health records. Comput Inform Nurs 2017;35:329-30.

19. DeVoe JE, Gold R, Cottrell E, et al. The ADVANCE network: accelerating data value across a national community health center network. J Am Med Inform Assoc 2014;21:591-5.

20. National Association of Community Health Centers. A sketch of community health centers, chart book 2014. Bethesda, M.D, 2014.

21. Peng M, Chen G, Kaplan GG, et al. Methods of defining hypertension in electronic medical records: validation against national survey data. $J$ Public Health 2016;38:e392-e399.

22. Gold R, Bailey SR, O'Malley JP, et al. Estimating demand for care after a medicaid expansion: lessons from Oregon. J Ambul Care Manage 2014;37:282-92.

23. NCQA. HEDIS 2016. 2016 http://www.ncqa.org/HEDISQualityMeas urement/HEDISMeasures/HEDIS2016.aspx (Accessed 12 May 2016).
24. Green BB, Anderson ML, Cook AJ, et al. Using body mass index data in the electronic health record to calculate cardiovascular risk. Am J Prev Med 2012;42:342-7.

25. Bazemore AW, Cottrell EK, Gold R, et al. "Community vital signs": incorporating geocoded social determinants into electronic records to promote patient and population health. J Am Med Inform Assoc 2016;23:407-12.

26. Lechner M. The estimation of causal effects by difference-indifference methods. Foundations and Trends in Econometrics 2011:4:165-224.

27. Dean CB, Nielsen JD. Generalized linear mixed models: a review and some extensions. Lifetime Data Anal 2007;13:497-512.

28. Teachman J. Latent growth curve models with random and fixed effects: Emerging Methods in Family Research: Springer International Publishing, 2014:3-17.

29. Stuart EA, Huskamp HA, Duckworth $\mathrm{K}$, et al. Using propensity scores in difference-in-differences models to estimate the effects of a policy change. Health Serv Outcomes Res Methodol 2014;14:166-82.

30. Hoffman C, Paradise J. Health insurance and access to health care in the United States. Ann N Y Acad Sci 2008;1136:149-60.

31. Baicker K, Taubman SL, Allen HL, et al. The Oregon experiment-effects of Medicaid on clinical outcomes. N Engl J Med 2013:368:1713-22.

32. Decker SL. Two-thirds of primary care physicians accepted new Medicaid patients in 2011-12: a baseline to measure future acceptance rates. Health Aff 2013;32:1183-7.

33. Saloner B, Kenney G, Polsky D, et al. The availability of new patient appointments for primary care at Federally Qualified Health Centers: findings from an audit study. 2014.

34. Little TD, Schnabel KU, Baumert J. Modeling longitudinal and multilevel data 2000. New Jersey: Lawrence Earlbaum Associates, 2000.

35. Zhang Z, Fang $\mathrm{H}$, Wang $\mathrm{H}$. Multiple Imputation based Clustering Validation (MIV) for big longitudinal trial data with missing values in eHealth. J Med Syst 2016;40:146.

36. Butler DC, Petterson S, Phillips RL, et al. Measures of social deprivation that predict health care access and need within a rational area of primary care service delivery. Health Serv Res 2013;48(2 Pt 1):539-59. 